\title{
Une approche des rapports entre pédagogie et science dans Les Cahiers pédagogiques entre 1945 et 1968
}

An approach about the relationship between education and science in Les

Cahiers pédagogiques between 1945 and 1968

\section{Xavier Riondet}

\section{OpenEdition \\ Journals}

Édition électronique

URL : https://journals.openedition.org/educationdidactique/1787

DOI : 10.4000/educationdidactique. 1787

ISSN : 2111-4838

Éditeur

Presses universitaires de Rennes

\section{Édition imprimée}

Date de publication : 31 décembre 2013

ISBN : 978-2-7535-3327-1

ISSN : 1956-3485

Référence électronique

Xavier Riondet, «Une approche des rapports entre pédagogie et science dans Les Cahiers pédagogiques entre 1945 et $1968 »$, Éducation et didactique [En ligne], 7-3 | 2013, mis en ligne le 31 décembre 2015, consulté le 16 août 2022. URL : http://journals.openedition.org/educationdidactique/ 1787 ; DOI : https://doi.org/10.4000/educationdidactique.1787 


\section{UNE APPROCHE DES RAPPORTS ENTRE PÉDAGOGIE ET SCIENCE DANS LES CAHIERS PÉDAGOGIQUES ENTRE 1945 ET 1968}

Xavier Riondet

Le propos de cet article est de comprendre les raisons de l'inertie du modèle disciplinaire de l'histoire scolaire qui produit, à l'école primaire et dans le secondaire, un enseignement visant surtout la mémorisation et la restitution par les élèves de données factuelles. L'analyse d'une leçon d'histoire au Cours Moyen portant sur l'empire de Charlemagne, 'objet de cet article est la question des rapports entre pédagogie et science à travers le corpus historique de la revue Les Cahiers pédagogiques entre 1945 et 1968. En étudiant la spécificité de cette revue, de ses contributeurs et de leurs discours (mais également de leurs pratiques éducatives), il s'agit de réfléchir au statut du pédagogue à l'œuvre dans ce corpus et à son rapport à la science. Lhypothèse de départ est liée aux partages conceptuels de Durkheim au sujet des termes "éducation», "pédagogie » et " science de l'éducation ». En mobilisant les données historiques du corpus étudié en dépassant l'opposition entre "pédagogie » et " didactique », l'enjeu est de contribuer à une histoire des expérimentations didactiques en marge du développement des sciences en s'appuyant sur une approche théorique originale.

Mots-clés : Histoire, Les Cahiers pédagogiques, pédagogie, science, didactique

An approach about the relationship between education and science in Les Cahiers pédagogiques between 1945 and 1968

The purpose of this article is the question of the relationship between education and science through the historical corpus of the review Les Cahiers pédagogiques between 1945 and 1968. By studying the specificity of this review, its contributors and their speech (but also their educational practices), I propose a reflection about the status of the teacher in this corpus and its relationship to science. The assumption is linked to conceptual shares proposed by Durkheim about the terms «education», "educational theory» and "science education». Mobilizing this historical corpus, beyond the opposition between "educational theory» and "didactic», the challenge is to contribute to a history of didactic experiments on the sidelines of the development of science based on an original theoretical approach.

Keywords: History, Les Cahiers pédagogiques, science, didactic. 
Cet article s'inscrit dans une approche historique visant à montrer l'intérêt que porte la problématisation novatrice de l'éducation entre 1945 et 1968, dans Les Cahiers pédagogiques, à la question d'une possible scientificité de la pédagogie. Une réflexion au sujet d'une réforme d'envergure en éducation, s'inaugure en 1945 sur fond de reconstruction de la nation aux lendemains de la Seconde Guerre Mondiale ; à l'autre extrémité de notre périodisation, l'année 1968 est d'une certaine manière le point d'orgue d'une vingtaine d'années de débats, de réflexions et de propositions sur l'École, sur fond de grande crise de société.

La revue apparaît dans le sillon de la commission Langevin-Wallon, nommée en 1944 par René Capitant. Dans l'attente du Plan Langevin, Gustave Monod, Directeur de l'enseignement du Second degré, annonce l'ouverture de classes d'expérimentation, dites classes nouvelles, afin de mettre en ouvre les réflexions du moment ${ }^{1}$. La revue des Cahiers devient alors le bulletin de liaison des enseignants de ces classes nouvelles localisées dans la région Rhône-Alpes. Si le plan Langevin n'est finalement pas adopté, la revue continuera de publier en se modifiant : en 1948, la revue prend le titre de Cahiers pédagogiques pour l'enseignement du Second degré dans l'optique d'élargir les questions et le lectorat des classes nouvelles au Second degré. Si bien qu'en 1952, lorsque les classes nouvelles disparaissent, la revue perdure, avec un même réseau de personnes et d'auteurs, en se focalisant sur les questions du Second degré, mais également sur les questions éducatives en général.

Dans ce contexte, la question est celle de la recherche d'une voie pour apporter une dimension scientifique à la pédagogie, reliée à une " problématisation $^{2}$ » de l'éducation. C'est en effet cette question qui va singulariser profondément la pensée des Cahiers dans cette période. La démarche de recherche va consister à extraire une cohérence et décrire un «style de pensée » (Fleck, 2005), à partir de fragments de textes divers qui eux-mêmes reposent sur un certain nombre de conditions d'existence et de possibilité. À partir de cette démarche, l'enjeu est de proposer une réflexion sur le statut du pédagogue par rapport aux questions éducatives et son rapport à la science.

\section{PÉDAGOGIE ET SCIENCE : DE DURKHEIM AUX CAHIERS PÉDAGOGIQUES}

Notre étude s'origine dans la remise en question d'une conception de la pédagogie comme un ensemble unitaire, occupant une place délimitée par rapport aux sciences de l'éducation, et par rapport à ce qui est devenu la didactique. L'enjeu est double : d'un point de vue historique, cela demande de porter un regard sur les archives mobilisées sans catégorisation a priori ; d'un point de vue philosophique, il s'agit d'envisager une alternative à un « ordre du discours " (Foucault, 1971) où la pédagogie semble rivale de la didactique dans un contexte particulier, les sciences de l'éducation.

\section{La pédagogie mise en discussion}

À l'occasion d'un débat entre Kambouchner et Meirieu publié dans la Revue Française de Pédagogie en $2001^{3}$, Meirieu parle d'une « pensée pédagogique, unitaire et cohérente », et précise qu' " [il la ferait] remonter à l'expérience de Pestalozzi » (p. 6). Selon lui, cette pensée pédagogique se situerait entre, d'une part, des « discours généraux et généreux sur l'épanouissement de l'enfant », et d'autre part, des "prescriptions technocratiques». Meirieu définit cette pensée comme " un discours qui s'installe dans les contradictions vives de l'acte éducatif et aide à les penser, à les dépasser par une action, non point contrôlée par une institution vétilleuse, mais régulée par des hommes et des femmes qui travaillent dans et sur des situations complexes » (p. 6-7). Cet ensemble hétérogène se trouverait depuis quelques années marginalisé au sein même des sciences de l'éducation car ces dernières « [seraient] aujourd'hui largement aux mains des didacticiens et des sociologues, après avoir été dominées, à l'origine, par les psychologues $»$. La pédagogie se retrouverait donc aux prises avec deux conflits liés entre eux, mais d'échelle différente : les rapports entre pédagogie et sciences de l'éducation d'une part ; les rapports entre pédagogie et didactique d'autre part.

L'émergence des sciences de l'éducation en 1967 est un premier élément historique pour expliquer cette marginalisation supposée de la pédagogie. Historiquement, lors des premiers cours de science de l'éducation à la fin du XIX ${ }^{e}$ siècle, la pédagogie s'enseignait à partir d'une culture regroupant auteurs 
et œuvres, à travers des leçons professées majoritairement par des philosophes. L'institutionnalisation des sciences de l'éducation en 1967 témoignerait d'une situation différente (Houssaye, 2009) : les philosophes et la philosophie de l'éducation seraient marginalisés.

Le second élément est contemporain du précédent. Lorsqu'en 1996, Astolfi et Houssaye débattent, entre autres, sur les similarités et les différences entre didactique et pédagogie, le premier cité explique ceci :

« Le fond du débat est épistémologique, et on sait qu'il n'y a pas de vérité de l'épistémologie. Seulement des positions. En définitive, il ne s'agit pas tant de savoir s'il est vrai (ou non) que didactique et pédagogie sont superposables que de savoir ce qu'on gagne (ou ce qu'on perd) à le prétendre et à le discuter» (Astolfi et Houssaye, 1996, p. 21).

On peut penser que le fond du débat n'est pas épistémologique, mais qu'il s'agit plutôt d'une reconstruction théorique des enjeux de l'enseignement opérée dans les années 70 , avec les premières publications de Brousseau et la création du Centre de Recherche et d'Observation sur l'Enseignement des Mathématiques, à l'école Jules Michelet de Tallence ${ }^{4}$ (Marchive, 2008, p. 67) où Brousseau a élaboré sa théorie des situations didactiques. Il est historiquement difficile de ne pas envisager ses travaux comme une révolution qui a ouvert la voie aux autres didactiques disciplinaires, et s'est imposée au sein même des sciences de l'éducation, en absorbant la tradition pédagogique.

\section{Pédagogie et science dans les Cahiers}

Quelle place occupent alors les militants des Cahiers, et surtout, qu'en est-il des rapports entre pédagogie et science dans le corpus étudié ? Une hypothèse provisoire serait d'influence durkheimienne : la réflexion pédagogique se déploie à partir de connaissances, d'apports scientifiques, et constitue un jugement sur l'éducation.

Lorsqu'il s'agit de définir spécifiquement la pédagogie, l'ouvrage Éducation et sociologie (2006) de Durkheim apparaît incontournable. L'éclairage apporté par Durkheim permet en effet d'opérer deux types de distinction : entre éducation et pédagogie, et entre science de l'éducation et pédagogie. L'éducation est un « fait continu », et la pédagogie, un « fait intermittent ». Durkheim explique qu' " il est vain de croire que nous pouvons élever nos enfants comme nous voulons » (Durkheim, 2006, p. 45). L'éducation est un « fait social ${ }^{5}$ et la pédagogie une «théorie pratique [...] [qui] réfléchit en vue de fournir à l'activité de l'éducateur des idées qui le dirigent » (ibid., p. 79).

Ce qui distingue " réflexions pédagogiques » et « science de l'éducation » repose sur la différence entre discours sur ce qui est et discours au sujet de ce qui doit être. D'une part, la science de l'éducation doit s'articuler autour de la formation des enseignants et à partir d'autres sciences (sociologie, psychologie). D'autre part, dans ce cadre, la réflexion pédagogique consiste en la formulation d'un jugement, à partir de connaissances parfaitement délimitées, sur des pratiques devenues routinières. La pédagogie permet donc au processus de l'éducation de se réadapter par rapport à un dysfonctionnement car la pédagogie s'appuie sur la science, en même temps qu'elle la questionne, l'interpelle ${ }^{6}$.

Cependant, le partage conceptuel durkheimien renvoie à un contexte bien différent de celui qui nous intéresse ici : de 1945 à la création des sciences de l'éducation et jusqu'en 1968. Il est alors intéressant de se demander comment se situent certaines voix méconnues de la pédagogie dans cet espace intermédiaire : s'inscrivent-elles dans le partage conceptuel de Durkheim ? Quels liens entre pédagogie et science peut-on observer dans leur pensée? Au sujet des questions de pédagogie, de didactique et de science, retrouve-t-on les clivages et les tensions dont parle Meirieu ? Les partages conceptuels de Durkheim au sujet de "pédagogie », "éducation » et « science de l'éducation » vont donc constituer dans notre étude une première série de repères. Ils nous fourniront des jalons pour réfléchir aux rapports entre pédagogie et science dans le corpus des premières publications des Cahiers.

\section{Particularité du corpus}

Nous pourrions dire des Cahiers qu'ils sont à la fois une revue, un mouvement pédagogique, un outil de formation professionnelle, un espace hétérogène de débat et de questionnement, un espace de circulation de savoirs (y compris scientifiques). Il s'agit en premier lieu d'un carrefour d'expérimentation 
de praticiens, fortement relié à l'objectif des classes nouvelles de donner une "formation complète » aux élèves:

«Les classes nouvelles sont des classes de $6^{\mathrm{e}}$, de $5^{\mathrm{e}}$, de $4^{\mathrm{e}}$, des lycées et collèges classiques, techniques et modernes ; elles ont le même programme que les autres classes et conduisent aux mêmes examens; mais elles sont organisées d'une manière spéciale afin de donner une orientation plus méthodique et une formation plus complète. (...) Les classes nouvelles visent non seulement à instruire, mais à éduquer, à former, outre l'intelligence, le caractère, l'affectivité, le sens social et toutes les qualités de l'être » (Pol-Simon et Goblot, 1947 , p. 13).

Bien que les méthodes nouvelles aient toujours été utilisées par une minorité d'enseignants, la revue apparaît en parallèle comme un outil de co-formation professionnelle. La spécificité originelle des Cahiers est effectivement de suppléer « à la carence de l'Éducation nationale en matière de formation initiale des maîtres » (Les Cahiers pédagogiques, 1972, p. 2) ${ }^{7}$. La revue se présente comme un lieu de circulation de savoirs, y compris scientifiques : certains universitaires y contribuent ponctuellement; parfois même, la revue publie les retranscriptions des conférences universitaires prononcées lors des stages de formation ; d'autres textes d'enseignants présentent régulièrement des pédagogues " historiques », souvent oubliés (Kerschensteiner, Jacotot et bien d'autres), des comptes rendus de lecture, des recherches de terrain, des exemples d'expérimentations suivant un protocole méthodologique.

Pour recueillir des données sur cette question historique des rapports entre pédagogie et sciences dans les Cahiers, nous choisissons d'envisager cette revue et ses marges comme un « collectif de pensée ». Nous prenons le collectif de pensée comme paradigme d'une « communauté [de] personnes qui échangent des idées ou qui interagissent intellectuellement (Fleck, 2005, p. 74). En suivant le raisonnement de Douglas (1999), nous pourrions envisager de parler de "monde de pensée ». Ce "monde de pensée » assignerait " aux objets du monde qui les unissent une identité et une fonction précises » (Sensevy, 2011, p. 93) ${ }^{8}$.

L'avantage de ces concepts est de rendre compte de l'impact de ces échanges et de ce qui constitue ces réseaux dans l'émergence d'un domaine de connais- sance. Il permet de saisir ce qui émane de ces relations sans pour autant former de grandes catégories interprétatives générales. De ce collectif de pensée se déduit un style de pensée identifiable dans ces réseaux, reposant sur des « connexions passives contraignantes » qui constituent ici la « consistance logique » du style de pensée à l'œuvre (Sensevy, 2001, p. 117). Dans le cadre de ses recherches sur Binet, Alexandre Klein (2008) mobilise ces concepts de « collectif de pensée » et de «style de pensée ». Selon Klein, l'histoire de la correspondance permet la description d'un collectif de pensée et l'émergence d'un style de pensée à l'œuvre. L'utilisation de ce concept comme grille de lecture d'un réseau intellectuel est conditionnée par deux postulats : d'une part, l'idée empruntée à Canguilhem d'une « histoire des sciences de la non-scientificité de son objet $»$ (Klein, 2008 , p. 26) ${ }^{9}$; et d'autre part, la conception d'une étude des échanges et des réseaux comme "outil d'une épistémologie philosophique » rendant compte d' « une recherche de la vérité » (id.) ${ }^{10}$.

Notre ambition est d'initier un programme de recherche similaire concernant les réseaux de militants pédagogiques en toile de fond de l'émergence du champ scientifique des sciences de l'éducation et en marge de l'histoire des didactiques scolaires.

\section{LES CAHIERS PÉdAgOGIQUES COMME COLLECTIF DE PENSÉE}

L'histoire des Cahiers repose sur plusieurs cercles, plus ou moins distincts, qui rentrent en interaction en de multiples circonstances, autour de nombreux objets distincts.

\section{Des cercles concentriques}

Un premier cercle constitue le cœur de l' « esprit des Cahiers », il s'agit des enseignants des classes nouvelles gravitant autour de la figure de François Goblot (1904-1974) et des membres de l'ANECNES (Association Nationale des Enseignants des Classes Nouvelles de l'Enseignement Secondaire). Fils du philosophe Edmond Goblot, Goblot décide en 1945 de créer un bulletin d'informations. L'ancrage lyonnais est important à saisir. En 1945, Goblot devient chargé de mission pour les classes nouvelles au lycée du Parc à Lyon. Il souhaite faire connaître les tenta- 
tives d'expérimentation du réseau lyonnais. Dans le cadre de sa mission, Goblot est en lien avec l'École Pratique de Psychologie et de Pédagogie du philosophe Jean Bourjade. C'est notamment grâce à cet institut d'université que la revue Les Dossiers pédagogiques pour l'enseignement du Second degré (format qui devient en 1948 Les Cahiers pédagogiques pour l'enseignement du Second degré) peut paraître. Goblot signe la majorité des textes lors de la première année de publication. La publication comprend initialement des témoignages d'expérimentations en classes nouvelles principalement situées en Rhône-Alpes. Le réseau s'élargit à partir de 1946, date de la création de l'ANECNES, association qui tente de fédérer les enseignants des classes nouvelles, puis toute personne proche des classes nouvelles, de manière à former à la fois des sections locales, qui travaillent en coopération, et une représentation plus nationale, pour échanger avec les hiérarchies. D’année en année, le réseau occupe tout le territoire français; les grands pôles sont notamment: Biarritz, Marseilleveyre, Sèvres, Montgeron, Toulouse (Savoye, 2010, p. 60).

Un deuxième cercle gravite autour de ce premier cercle : il s'agit du réseau administratif autour des classes nouvelles, comme les conseillers techniques pédagogiques et les conseillers pédagogiques, et dont le point névralgique est constitué de la Direction du Second degré de 1945 à 1950, dont la figure historique est Gustave Monod (1885-1968). Ce deuxième cercle se constitue prioritairement des relations et échanges qui animaient l'étage de la Direction du second degré rue de Grenelle, à Paris ${ }^{11}$. Dans ce cercle, nous pouvons mentionner également les personnes très proches des classes nouvelles et qui intervenaient dans le cadre de la formation de ces éducateurs, nous faisons référence ici à Edmée Hatinguais (1896-1972), directrice du CIEP (Centre International d'Etudes Pédagogiques). Ce cercle s'occupe notamment de l'organisation des stages de formation organisés au CIEP et de coordonner localement les actions, les échanges et d'organiser certaines réunions ou rassemblements. Les stages de formation sont destinés aux chefs d'équipe de classes nouvelles. Le déroulement se séquence autour de plusieurs moments : conférences de spécialistes, d'universitaires, de scientifiques ; conférences de collègues; ateliers, mise en commun; etc.

En périphérie de la revue, on trouve des milieux divers. Des universitaires tout d'abord : Maurice Debesse (1903-1998) intervient ponctuellement à plusieurs reprises ; d'autres, comme Gaston Mialaret, interviennent plus épisodiquement pour rendre hommage aux grandes figures disparues. Nous retrouvons également des inspecteurs, des recteurs, ainsi que des responsables d'organisation, de mouvement de jeunesse et d'institutions diverses (CUIP ${ }^{12}$, $\mathrm{IPN}^{13}$, etc.). Rappelons également que nous pouvons lire, dans la revue, des contributions extérieures et des retranscriptions de conférences, provenant de stages des classes nouvelles, ou d'autres contextes. C'est le cas notamment d'une conférence du philosophe Gaston Bachelard (1884-1962) et d'un article de l'économiste Jean Fourastié (1907-1990).

\section{À travers le format de la revue, des réseaux complexes}

La forme de la revue et son évolution permettent de vérifier la présence de ces cercles et leurs interactions. Par exemple, Octave Leclancher (1907-1949), professeur de lettres au lycée du Parc à Lyon, dans l'établissement où Goblot est chargé de mission pour les classes nouvelles, prend en charge la rubrique de l' « Homme à l'oreille cassée », une rubrique qui fait état de ce qui s'est dit dans les quotidiens, les revues pédagogiques, ou les revues d'association d'agrégés. De son côté, Claire Roby (1892-1974), conseillère pédagogique, est l'auteure de contributions très importantes, au même titre que Suzanne Brunet, dont les écrits dans la revue ont particulièrement influencés les enseignants des classes nouvelles. De même, Fernand Gaillard, enseignant de Dijon, contribue aux regroupements de Sèvres en étant le spécialiste historique des figures historiques. Il profite de la revue pour présenter à plusieurs reprises des figures oubliées de la pédagogie, mais également présenter une enquête sur la question de l'interrogation.

Nous pouvons distinguer la complexité des réseaux qui traversent la revue, en ajoutant que plusieurs contributeurs appartiennent à plusieurs cercles simultanément. Alfred Weiler (1901-1961) est un exemple intéressant. Lorsqu'Alfred Weiler dirige l'annexe de Montgeron en 1946, ce dernier est déjà une figure bien connue de l'Éducation nouvelle. Outre sa participation à l'équipe de Monod, Weiler est en charge avec Marcel-Etienne Ginat (1897-1937) du Congrès du Havre de 1936 (Condette et Savoye, 2011). 
D'autres contributeurs, comme Lucien Lefèvre, ont la particularité de progresser de cercle en cercle. Lucien (Étienne) Lefèvre, est né en 1912. Après des études en Histoire et Géographie, il enseigne en Lycée de 1936 à 1968, et en particulier dans les classes nouvelles. Docteur en pédagogie en 1964, il poursuit sa carrière en Sciences de l'éducation et à l'INRDP (Éducation et prospective, p. 140). Louis Cros (1908-2000) est un autre exemple très intéressant : inspecteur, puis en poste au ministère, il permet, via le CUIP, de publier la revue de 1954 à 1962 et il y contribue tout en publiant un ouvrage majeur, L'explosion scolaire (1961).

\section{RECONSTITUTION DU STYLE DE PENSÉE DES CAHIERS}

Par « style de pensée », nous pouvons envisager la question d' " un commun voir-comme » (Sensevy, 2011, p. 46). Trois idées sont particulièrement représentatives du collectif étudié : l'articulation principe/ pratique ; la connaissance de l'enfant ; la question du milieu.

\section{Larticulation entre les principes et les pratiques}

Dans l'optique des Cahiers, la spécificité du style de pensée éducative repose sur trois principes philosophiques : en éducation, il s'agit de rendre possible la liberté de l'autre (l'élève); cette liberté consiste en une articulation entre autonomie du jugement et appréhension de l'avenir ; enfin, la valeur de l'authenticité est vue comme une condition de possibilité et une finalité en soi. C'est ainsi que l'éducation, envisagée ici en tant qu'apprentissage de la vie, apparaît comme devant permettre à l'autre (l'élève), d' « être quelqu'un » qui sera «le même avec ses amis, sa famille, ses compagnons de travail, chefs et subordonnés » (Goblot, 1945a, p. 594).

Loin d'être simplement une philosophie spéculative, ces idées animées par une volonté manifeste d'articuler concrètement Éducation nationale et Éducation nouvelle, sont centrales dans la revue (Weiler, 1950, et Goblot, 1950). L'ombre du raisonnement de logicien ne fait ici aucun doute :

«Dans les traités sur l'éducation nouvelle, on trouve souvent, dans un premier chapitre, un exposé des grands principes : spontanéité, activité fonctionnelle, tendances, autonomie, etc. Mais au $\mathrm{n}^{\text {ième }}$ chapitre l'auteur éprouve le besoin d'une mise au point, il craint qu'on n'aille trop loin, comme si les principes énoncés au début ne couvraient pas toute la réalité, comme si on s'avisait tout d'un coup qu'une certaine forme idéale de l'éducation nouvelle vient se heurter à une obscure puissance anarchique de la matière: le pédagogue démiurge semble pris d'un remords devant le monde qui sort de ses mains. Remords inquiétant pour le logicien, car enfin ou bien on sait que les principes du début sont bons pour les avoir expérimentés et jugés à leurs résultats, ou ces principes ne sont pas suffisants pour régler notre conduite et alors il serait honnête de le dire tout de suite et d'expliquer pourquoi ; il serait sage de les compléter » (Goblot, 1950, p. 190).

Les classes nouvelles se présentent comme une application des principes de l'Éducation nouvelle à l'enseignement secondaire français (Bloch, 1953, p. VI). L'ambition initiale est de rompre avec l'encyclopédisme dissymétrique pour faire émerger une culture humaine, individualiste et sociale : si l'objectif est de développer la coopération et de faire de du milieu scolaire " une communauté de travail amicale et vivante », il est aussi question de «porter attention à la vocation personnelle des enfants » (ibid., p. 12). Mais la grande caractéristique de ces classes nouvelles est « de soumettre à l'épreuve de la pratique, d'expérimenter dans le cadre de «l'institution » nouvelle de nouvelles méthodes d'enseignement ou d'éducation» (ibid., p. 3). Ce qui engendre une grande hétérogénéité dans le déroulement: accent mis sur l'individualisation, sur les travaux d'équipes, sur l'étude du milieu et/ou la création d'une communauté scolaire. Les classes nouvelles ne renvoient donc pas à une « collection définie de procédés ou de recettes », mais peuvent se définir comme un « effort de rénovation en profondeur [de] la pédagogie du second degré » pouvant « se déployer en une multitude de directions » (ibid., p. 7).

Ces méthodes nouvelles ont la particularité d'être articulées autour d'un travail de connaissance de la psychologie de l'enfant et de l'adolescent et d'une approche du milieu. L'ensemble des publications (Dossiers, Cahiers, Bulletin) fournit des réflexions et des outils à utiliser ${ }^{14}$. Le fait que plusieurs enseignants de ce réseau aient publié un ouvrage par ailleurs (Lefèvre, 1949 ; Zadou-Naïsky, 1954) permet d'illustrer cet apport de la revue et l'utilisation qu'en ont fait certains enseignants au quotidien. 
L'éducateur et la connaissance de l'enfant : « chaque enfant pose un problème particulier »

Lucien Lefèvre publie en 1949 une description et une analyse de sa démarche pédagogique en classes nouvelles à partir des données, qui ont la particularité d'avoir été parfois fournies par la revue. Durant ses quatre premières années d'enseignement, Lefèvre enseigne entre Étampes et le lycée de Montgeron. La particularité de ce dernier établissement est d'être l'annexe du Lycée Henri IV de Paris et d'avoir été créé spécifiquement pour l'ouverture de classes nouvelles. Le proviseur de ce lycée est un certain Alfred Weiler. L'objet du livre est « de voir comment un maître a été amené de plus en plus à s'orienter vers des préoccupations psychologiques dans une classe nouvelle, comment il a adapté son enseignement à ces préoccupations et quelles suggestions lui a inspirées son expérience » (Lefèvre, 1949, p. 9).

La connaissance de l'enfant est entreprise par l'équipe de Lefèvre à partir d'une double-enquête reposant sur des tests (individuels, collectifs) et des observations. C'est ici la posture des professeurs et autres membres de l'établissement qui est à souligner : ils confrontent les résultats, réajustent les activités en continuant de recueillir des données, en mêlant éventuellement les parents.

\section{L'enseignant et la question du milieu}

Le nom d'Alfred Weiler est resté associé à la question de l'étude du milieu. Nul n'a davantage habité cette question que Weiler, et celle-ci illustrait parfaitement les principaux traits de sa pensée pédagogique. Selon lui, l'enseignement se doit de « donner une culture riche, souple et vivante " (Chambon, Delchet et Lefèvre, 1974, p. 374). L'éducation se doit être en lien avec la vie et son objectif doit être de «préparer à la vie » $(i d .)^{15}$. En ce sens, l'étude du milieu constitue la pièce centrale et complexe de la pensée de Weiler. Si l'intérêt des enfants doit être suscité à partir d'une dialectique "dépaysement/ environnement », la finalité est bien de travailler à l'émergence d'un « esprit autonome » progressif, démocrate et humaniste. L'éducation devant lutter contre l'égoïsme, le dogmatisme et le conformisme, la classe est donc envisagée depuis un climat de confiance. C'est également le cas au niveau de l'établissement à partir d'un système d'autodiscipline.
Pour Weiler, "Il s'agit moins en réalité d'une étude $d u$ milieu, que de diverses études ayant pour objets des milieux variés, différenciés, enchevêtrés » (1949, p. 8). Cela induit une grande hétérogénéité des types de milieu : " il comprend tous les cadres de notre vie : milieu naturel, biologique, milieu humain, familial, scolaire, etc. ». L'étude du milieu est tournée vers la synthèse, tout en faisant jouer le détail face au global. L'enjeu est de remédier au « fatras livresque » de l'école et de « retrouver l'unité de la connaissance ».

Il ne s'agit pas d' " ouvrir l'école sur la vie », mais de «nourrir l'école de la substance de la vie ». Par l'étude du milieu doivent être mis en lumière les prolongements possibles et « l'étroite solidarité avec un ensemble plus vaste ». C'est au niveau de cette " solidarité » qu'intervient l'éducateur car l' "avantage inestimable » dans l'étude du milieu est que « le professeur est maître de son destin ». Le génie de l'éducateur est donc de penser une situation d'étude du milieu où il est possible de « s'élever à un point de vue d'où l'on dépasse les spécialités pour apercevoir leurs connexions dans un complexe général ».

Létude du milieu après les classes nouvelles : l'exemple du « tourisme éducatif » en 1958

Le dossier " tourisme et enseignement ${ }^{16}$ publié en 1958 témoigne de l'influence du réseau des classes nouvelles après leur disparition institutionnelle à partir de 1952. Ce court dossier se compose d'un entretien avec trois enseignants : Paul Chavanne, professeur de lettres ; Louis Bois, professeur de lettres également ; et Henri Mérignon, professeur d'allemand; tous trois professeurs au Lycée Marseilleveyre, établissement qui a été créé dans le cadre des classes nouvelles ${ }^{17}$. Le dossier montre le souci d'adaptation des situations d'enseignement à un phénomène de société (en l'occurrence, le tourisme) tout en gardant à l'esprit la finalité de découvrir une autre culture, une histoire, des problématiques, et de se confronter avec la connaissance (notamment concernant les disciplines de l'Histoire et de la Géographie). Merignon est alors parfaitement conscient que « le rôle de l'école n'est pas de faire du tourisme ; il est d'organiser des voyages qui soient assez différents du tourisme tel qu'on le conçoit trop souvent : visite de Paris la nuit, à 10 heures 15 on quitte telle boîte, à 10 heures 25 on arrive dans telle 
autre...» (Bois, Chavanne et Merignon, 1958, p. 14). L'école doit former, préparer à un tourisme intellectuel. Prenons l'exemple d'un voyage en Grèce ${ }^{18}$. Le voyage constituait l'occasion pour les enfants de trouver là-bas « un pays vivant » (ibid., p. 13), la Grèce de la fin des années 50. À l'aller, dans le bateau, les élèves ont ainsi procédé à des entretiens avec les enseignants qui leur parlèrent de l'histoire de la Grèce, de la Grèce moderne, de la Grèce classique, et des différentes formes d'art et d'architecture que l'on peut y rencontrer. Si la motivation pour ce voyage était liée à un intérêt, déjà là, pour la Grèce, cet intérêt s'est entretenu à partir des " choses vues ». $\mathrm{Au}$ retour, le mouvement était inverse, les élèves avec les enseignants ont fait le bilan : confronter les impressions ; rafraîchir les souvenirs. Les enseignants soulignent le double apport du voyage : culturel et éducatif. Apport culturel, par la découverte active de la Grèce, mais également, apport éducatif ; les enseignants insistent sur la découverte de chacun ${ }^{19}$.

Le milieu, la connaissance, la démocratie : réflexions sur l'éducation à la citoyenneté en 1959

Cette question de l'étude du milieu dans la période post-classes nouvelles comprend une approche de la citoyenneté très singulière. Au sujet de l'éducation à la citoyenneté, nous pouvons isoler deux tendances. La première est historiquement liée à l'enseignement traditionnel (avec une pédagogie dissymétrique et un contenu très encyclopédique) dans lequel existait l'instruction civique. Une autre tendance plus contemporaine aspire à faire débattre les élèves sur des questions d'actualité (prédominance de l'oral sur l'écrit) et à leur laisser des libertés et des responsabilités (journaux lycéens, conseils lycéens, etc.) (Blais, Gauchet et Ottavi, 2002). En réalité, cette deuxième tendance avait été pensée par Freinet dès le début de sa carrière (décennie 1920). Ces deux tendances consacrent deux logiques différentes : avec la première, l'érudition et la conformité feront le citoyen ; avec la seconde, la conscience de l'actualité et la pratique de la responsabilité développeront le sens de la citoyenneté. L'éducation à la citoyenneté dans le contexte de l'étude du milieu n'appartient ni à l'une, ni à l'autre de ces tendances. L'idée de " citoyenneté » n'est pas encore surdéterminée par le cadre socio-économique européen, mais envisagée par la question de la démocratie et de la formation morale.

Lors d'un Congrès des Cahiers pédagogiques à Marseille le 17 avril 1958, alors que la question du civisme pratique est abordée, M. Merignon, professeur d'allemand au lycée Marseilleveyre explique le renversement que doit vivre la question du civisme à l'École :

«Quand on parle d'instruction civique, on recherche ce que l'enfant doit apprendre, et c'est très bien, mais on arrive aussi à un enseignement magistral, donné de l'extérieur à l'enfant. Je crois qu'il faudrait l'inverse [...]» (Congrès de Marseille, 1959, p. 38).

Les contributeurs, pour la plupart issus du réseau des classes nouvelles, envisagent une éducation à la citoyenneté par un civisme pratique, qui articulerait pratique de la liberté et exercice de la responsabilité. L'enjeu est donc d'importance pour l'éducateur par rapport à la société, comme l'explique Yvette Bouisseren, professeur de philosophie au lycée Marseilleveyre :

« S'ils prennent auprès de nous le goût de la liberté qui refuse également l'esclavage et l'anarchie, et s'ils savent la défendre mieux que nous n'avons su le faire, profitant de nos succès et surtout de nos erreurs, alors nous aurons respecté leur personne en leur donnant l'occasion de se réaliser $»(1959$, p. 61).

Des exemples concrets sont mis en évidence dans la revue. P. Chavanne, professeur au lycée Marseilleveyre, évoque la réorganisation des classes et des internats en fonction de la formation du citoyen (l'auto-discipline est un exemple important) (1959, p. 40). Pourtant, un constat semble partagé, « l'éducation pratique ne suffit pas ». Jean Delannoy, enseignant de Biarritz et coordonnateur du dossier sur l'éducation à la citoyenneté, écrit :

«Pas plus que les leçons des différentes disciplines, elle ne rendra inutile le cours d'instruction civique. Renoncer à tout enseignement théorique serait très dangereux. Léducation pratique donne des habitudes, un comportement. Elle ne décide pas des principes qui doivent éclairer ce comportement. On peut être dévoué, serviable, prêt au sacrifice, plein d'abnégation et d'héroïsme, et cela contre l'humanité et, finalement contre son pays [...]» $(1959$, p. 45). 
Dans cette problématique des « principes éclairants », réside en fait la question de l'orientation :

« [...] avant la guerre, en Allemagne, j'ai passé plusieurs heures dans un compartiment de chemin de fer avec un groupe de jeunesse hitlérienne. Ces garçons étaient vraiment très gentils entre eux, ils faisaient un voyage long et se débrouillaient remarquablement pour s'en épargner mutuellement les fatigues. Quelque chose d'excellent avait été acquis là, avec une erreur monstrueuse d'orientation » (id.).

Le civisme doit être orienté vers la question du monde. Pour Geneviève Legrand, il faut « écouter battre le cour du monde » (Legrand, 1959). Cette enseignante base son cours d'éducation civique sur des questions qui évoquent le monde ${ }^{20}$. Cette " écoute » orientera leur pratique. Cette question du monde est donc un élément primordial, même au-delà de la discipline de l'éducation civique.

La problématisation de l'éducation à la citoyenneté dans la revue prend en compte la question des savoirs. Depuis l'épisode des classes nouvelles (19451952), les contributeurs ne cessent de critiquer le fait que les enseignements reposent sur logique d'encyclopédisme liée à une surcharge des programmes, se traduisant par un gavage d'esprit et une indigestion intellectuelle (Masset, 1958, p. 37). Les réflexions sur l'enseignement de la géographie et de l'histoire témoignent d'une réflexion sur la situation éducative, via la question du milieu, dans une finalité de formation à la citoyenneté.

En février 1958, un dossier de la revue porte sur la géographie et son enseignement à rénover. André Massat, inspecteur de l'enseignement technique à Toulouse, écrit :

«On n'enseigne pas en effet la géographie, on doit s'efforcer de la faire redécouvrir par les élèves. Le savoir n'est jamais gratuit. On ne sait vraiment que ce qu'on est capable de retrouver, réinventer, de recréer par un effort personnel. Tout savoir doit être le résultat d'une conquête, d'une exploration, et non d'une communication verbale du professeur [...]» (1958, p. 59).

Il faut partir du concret : «[...] Seule s'impose et se conçoit une géographie fondée sur l'observation. Son enseignement rejoint celui de la leçon de choses [...] » (id.). Localiser, décrire, expliquer ; voilà la triple démarche envisagée par André Massat. L'étude du milieu est explicitement évoquée comme point de départ possible de la géographie. D'un côté, l'étude du milieu consiste en « une prise de contact global avec le milieu dans lequel vit l'élève, milieu familial, milieu scolaire, milieu naturel, milieu humain, dans le cadre de la commune ou de la région, et qui a pour but d'amener l'enfant à prendre conscience du monde qui l'environne [...] » (Lefèvre, 1958, p. 68). De l'autre, la géographie est « une science constituée, avec ses lois, ses méthodes, ses ambitions propres

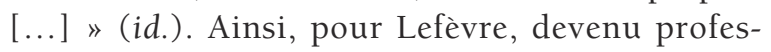
seur au Centre de rééducation de Garches, l'étude du milieu est un point de départ pour la leçon de géographie, ou un moyen de la rendre plus vivante. En géographie plus particulièrement, l'étude du milieu donne l'occasion de faire des comparaisons, de tirer des conclusions qui dépassent la géographie, mais éclairent d'un jour particulier les enseignements de celle-ci.

Les réflexions sur l'enseignement de l'histoire sont similaires. L'idée selon laquelle, l'élève doit se confronter à l'histoire, est très prégnante. L'élève n'est plus considéré comme un réceptacle qui reçoit, ingurgite, le savoir proféré magistralement par l'enseignant. L'enseignant a pour mission de confronter l'élève à un milieu. Une contribution de Gérard Westphal, professeur au collège moderne de Strasbourg est, à ce titre, particulièrement intéressante. En 1954, il fait part dans la revue d'une expérimentation : emmener ces élèves aux archives de la ville à l'occasion d'un cours sur la Révolution française, et faire procéder, par ses élèves de Troisième, à des recherches destinées à " rendre vivant le cours d'histoire » (Westphal, 1954, p. 174).

\section{Les enjeux et les difficultés de l'étude du milieu}

La réflexion de ces enseignants se déploie à partir d'un postulat de dissociation des volontés et de distinction des actions « enseigner » et « apprendre » sans nécessairement les disjoindre. Aujourd'hui, nous dirions avec Sensevy : "Enseigner c'est faire apprendre. Apprendre, c'est se faire enseigner. Même des situations qui semblent contester ces énoncés tombent sous leur régime. Le Professeur le plus émancipateur, dès lors qu'il aura l'intention que des Élèves apprennent, fera quelque chose en vue d'atteindre ce but. L'Élève le plus autodidacte, dès 
lors qu'il voudra apprendre, trouvera des voix dont il s'autorisera pour aller dans telle ou telle situation » (Sensevy, 2011, p. 642). Il s'agit ensuite d'envisager un milieu et de rendre possible la confrontation directe entre l'élève et ce milieu. Le raisonnement semble proche de ce que Sensevy explique en ces termes : « La manière concrète de dépasser la dépendance de l'élève au professeur et leur assujettissement au contrat didactique consiste ainsi à placer un milieu entre le professeur et l'élève. Ce que l'élève apprendra ne dépendra pas plus strictement de sa relation au professeur, mais de sa relation au milieu » (id.).

Ce raisonnement dépend de deux éléments. Nous sommes en présence d'une génération d'enseignants ayant vécu l'épisode de la Seconde guerre mondiale. Le traumatisme de phénomènes de domination qui ont marqué la première partie du $\mathrm{XX}^{\mathrm{e}}$ siècle, ainsi que le souvenir encore présent d'une société n'ayant pas nécessairement désobéi en certaines situations permettent de saisir cette volonté de ne pas faire de l'éducation, une entreprise de dogmatisme et de conformisme. De plus, l'École fait alors face à des phénomènes d'accélération (Riondet, 2010). Les éducateurs ne peuvent plus livrer un monde tel quel dont ils maîtriseraient toutes les techniques. L'éducation doit pouvoir permettre à l'élève de s'orienter dans le monde. Pour Fabre, le monde contemporain est un "monde problématique ", loin des "certitudes des sociétés traditionnelles", qui « requiert des repères plutôt que des chemins tracés » (Fabre, 2011, p. 7-8). C'est précisément sous la forme du "problème » que se joue l'enjeu et la difficulté de ces réflexions sur l'étude du milieu.

Il y a un rapport à l'actualité essentiel à mettre en situation, via la situation d'enseignement, pour permettre le lien entre un civisme pratique et la question du monde : il est nécessaire que le professeur procède à une actualisation de la question. S'il s'agit du sort des esclaves dans l'antiquité, on peut trouver des textes assez nombreux qui s'y rapportent, faire voir ce qu'était le problème du travail dans le monde antique, ce qu'il est dans le monde moderne. Le rapport à l'histoire (la recherche de l'historicité du monde) doit problématiser l'actualité, de manière à faire écho en l'élève et de laisser une possibilité d'action, par lui-même et pour lui-même, sur le monde. Le problème de l'élève doit également être un problème le reliant à la culture et au monde. Reprenant une rhétorique propre à Fabre (2011), nous pourrions dire que la boussole pour s'orienter n'a d'intérêt que reliée à une carte.

\section{LES RAPPORTS ENTRE PÉDAGOGIE ET SCIENCE(S)}

Tous ces éléments permettent de comprendre la portée d'un discours présentant la recherche et la réforme comme devant être guidée par une « foi démocratique » (terme emprunté à Dewey) et « une exigence de justice» (Monod, 1950, p. 4-5). S'enchevêtrent projet de réforme éducative, prise en compte de la dimension sociétale, et réflexions sur la pédagogie à la lumière et dans la dynamique du développement des sciences humaines et sociales.

Pour illustrer cela, nous pouvons prendre l'exemple du «plan d'ensemble » esquissé durant l'année 1950 dans la revue, qui s'inscrit dans le prolongement du plan Langevin ${ }^{21}$. Remis en 1947 , le Plan Langevin n'est pas appliqué, les militants des Cahiers ne cessent pourtant de réclamer la réforme scolaire tant attendue, qui serait en adéquation avec l'évolution de la société et qui prolongerait les résultats encourageants de l'expérience des classes nouvelles. À ce titre, lors de l'année 1950, la revue consacre deux dossiers au sujet de la réforme. Ces deux dossiers esquissent un "plan d'ensemble * qui peut permettre d'arbitrer la prochaine réforme que l'Administration va décider. Les divers protagonistes viennent d'horizons différents (le $\mathrm{BIE}^{22}$; l'université ; la direction du second degré). Certains, universitaires reconnus font partie de la périphérie de la revue ${ }^{23}$, d'autres forment le noyau même du mouvement des Cahiers. Le " plan d'ensemble » mobilise divers domaines et diverses spécialités en essayant d'aborder les choses avec la hauteur que peuvent offrir l'approche scientifique des problèmes et l'apport de connaissances spécifiques.

\section{Approche socio-économique des problématiques scolaires}

Dans un premier temps se manifeste la volonté de diagnostiquer scientifiquement le problème actuel de l'école française. Pedro Rossello, en tant que témoin privilégié des évolutions scolaires en Europe, décrit le phénomène de croissance quantitative de l'enseignement en général, et plus particulièrement de l'enseignement du Second degré. L'enseignement secondaire grandit, mais il change, il évolue. Il traverse une période d'instabilité ; ce qui crée d'ailleurs un certain malaise, écrit-il, car les Français semblaient convaincus que le pire était derrière 
eux. Ils ne distinguent pas quelle sera la prochain étape, quel sera le chantier qui va suivre. Ce n'est pas parce que l'enseignement secondaire se modifie que la clientèle augmente ; bien au contraire, c'est parce que la clientèle s'élargit que l'enseignement secondaire doit changer sa conception de l'organisation ; ce qui engendre diverses problématiques : l'orientation; les passages, les transitions ; l'accès à l'institution; la question des programmes et des méthodes. Cette prolongation de la scolarité résulte moins de l'action et de la volonté des figures de la pédagogie que « des besoins impérieux de l'industrialisation de notre monde et de la substitution de la machine à l'homme " (Rossello, 1950, p. 8). Rossello précise : «[...] c'est pour éviter le chômage que l'action en faveur de la prolongation a été déclenchée » (id.).

Cette idée est prolongée et détaillée depuis les sciences économiques et sociales par Jean Fourastié, professeur au Conservatoire national des Arts et Métiers. Selon lui, alors que la répartition de la population active en France en 1800 était la suivante : $80 \%$ dans le secteur primaire ; $10 \%$ dans le secteur secondaire ; $10 \%$ du secteur tertiaire ; la proportion a changé : un tiers dans chaque secteur. Le progrès technique, avec les changements qu'il a induits, a favorisé l'émergence de nouvelles crises, dont les répercussions ont pu s'observer sur les questions éducatives et les conditions d'enseignement : l'accroissement croissant des effectifs lié à l'évolution économique contemporaine et l'éloignement de l'École et de la vie. Pour Fourastié, l'extension des cadres matériels de l'enseignement et l'évolution de l'esprit de l'enseignement sont dès lors des domaines à explorer. Il met le doigt sur un point essentiel : «si le progrès technique est une cause de l'accroissement des effectifs scolaires, il faut bien comprendre qu'il est aussi un effet de la formation des jeunes générations; le progrès technique permet l'enseignement, mais un enseignement convenable est nécessaire à la poursuite du progrès. Non seulement la réduction croissante du travail physique implique un usage croissant du travail intellectuel, mais encore le développement du tertiaire implique plus spécialement l'initiation d'un nombre croissant de travailleurs aux mécanismes essentiels de la vie économique » (Fourastié, 1950, p. 18).

Deux problèmes sont ainsi posés :

- la civilisation économique contemporaine a libéré la jeunesse du « travail servile » et a ainsi ouvert à des masses sans cesse plus importantes la «possibilité matérielle » de recevoir une formation scolaire, secondaire puis supérieure ; l'École ne peut plus refermer ses portes, elle doit dorénavant « attirer » et « retenir » la jeunesse dans un cadre attrayant en proposant une formation utile (id.) ;

- la formation doit être faite pour l'homme moderne actuel, " tel que le fait le climat économique contemporain »; l'objectif est de former des beaux esprits, mais également des hommes d'action, équilibrés, qui auront à « remplir un rôle défini (agriculteurs, commerçants, ingénieurs...) dans une société complexe » (id.). Et c'est en l'occurrence une culture générale à base économique qui pourrait, selon Fourastié, permettre de répondre aux exigences du monde actuel puisque l'économie est la science du rapport des hommes aux choses, dit-il, et donc au carrefour des problèmes humains et sociaux.

\section{L'obstacle épistémologique et l'impératif humaniste}

L'approche socio-économique met donc particulièrement en évidence « l'obstacle épistémologique $^{24} »$ qui consiste à vouloir enseigner en 1950 comme cela se faisait à la fin du XIX ${ }^{\mathrm{e}}$ siècle, alors que le public et la société ont changé. Cela renvoie à la question de la configuration scolaire, comme n'étant plus adaptée au monde moderne. Charles Morazé (1913-2003), chargé de cours à l'Institut politique de l'Université de Paris, estime à cet effet que l'esprit de l'École des années 1950 est dépassé et depuis de longues années :

« [...] le fond de nos programmes d'enseignement secondaire, l'esprit qui préside à la répartition des heures de travail dans les lycées (mot grec), l'équilibre des examens, c'est une tradition antérieure à toutes les inventions qui ont bâti le monde moderne » (1950, p. 19).

Il en est de même pour Weiler, pour qui un enseignement correspond à une vision de l'humanité et à un contexte historique en particulier :

«Une réforme de l'enseignement traduit une conception de l'homme. Chaque grande époque de la civilisation se définit par une certaine forme d'humanisme et il en découle un système d'éducation. 
Jusqu'à présent, l'humanisme dont on a vécu notre enseignement est celui de la Renaissance. Notre époque appelle sans doute un humanisme, mais c'est un humanisme nouveau »(1950, p. 23-24).

Au sein de ces critiques sur la question de la configuration scolaire, il y a l'idée d'un humanisme dépassé. Cela ne signifie pas de considérer tout passé comme inutile. René Hubert (1885-1954), recteur de l'académie de Strasbourg, met en relief deux types de références au passé : la référence encombrante et dépassée et la référence vivifiante toujours apte à augmenter le présent. Pour penser ces deux types de passé, Hubert explique qu'il faut distinguer les traditions et les habitudes. Les habitudes sont des acquisitions individuelles (habitude de pensée ou de comportement) qui peuvent " prolonger la tradition, se superposer à elle, épouser sa forme comme un lit d'alluvions sur fond de roches dures » (Hubert, 1950, p. 27). La tradition, elle, est de l'ordre de la communauté, puisqu'étant « le système des représentations collectives [que le groupe social] reçoit de son passé » (id.). « [Les traditions], poursuit-il, sont le support social de la mentalité personnelle. De là ce qu'elles présentent apparemment de général et même d'abstrait : on dit les habitudes d'un éducateur, on dit les traditions des éducateurs, et, mieux encore, les traditions de l'enseignement, secondaire, primaire ou supérieur $»(i d$.$) .$

La tradition renvoie à des valeurs et des réalités socio-historiques qui ont influencé et modelé la configuration scolaire ${ }^{25}$. Ainsi, selon Hubert, il faut changer de références, de traditions, de postulats, et par la même occasion, que les éducateurs changent le cœur même de leurs habitudes. C'est-à-dire que les éducateurs doivent prendre conscience du sens et des références de leurs pratiques. Un nouvel esprit doit souffler sur l'École et traverser la conscience des éducateurs. Ce nouvel esprit, paradoxalement, est à puiser dans le passé ; voilà pourquoi Hubert propose de prolonger l'œuvre commencée il y a plusieurs siècles au nom de l'Éducation nouvelle.

Ce raisonnement fait écho aux propos de Gaston Bachelard (retranscrit dans la revue en 1950), évoquant la nécessité des connaissances mais également de l'esprit. S'il faut être en mesure de comprendre les mutations du progrès technique, la dimension de l'esprit est importante car, selon lui, " c'est l'école qui doit donner le modèle de société parfaite, car la société parfaite, c'est l'école »
(Bachelard, 1950, p. 30). C'est là, le dernier point essentiel du plan d'ensemble. Bachelard met deux choses en avant : l'homme instigateur de changement et l'école comme société parfaite. L'homme doit prendre la mesure de ses possibilités:

« Il faut que l'homme intervienne dans les choses et ait conscience de sa vertu de transformation ; intervenir, ce n'est pas faire à l'aveuglette des expériences pour voir. Nous sommes maintenant dans une époque où l'homme se désigne comme l'être des transformations » (id.).

Mais encore une fois, il ne s'agit pas de suivre ses instincts et de procéder à partir de ses propres impulsions ; la conscience de cette possibilité de transformation ne va pas sans un exercice préalable de la pensée : « [...] il faut qu'il y ait une pensée avant l'action, il faut rétablir la primauté de la connaissance, il faut avoir conscience de notre pouvoir de transformer le réel » (id.). Cette action de l'homme ne doit pas se faire sans un travail de la pensée, et ce travail doit être organisé par l'école et dans l'école: « [... ] L'école doit faire travailler la pensée, la mettre sur un plan de rationalité, et d'une rationalité qui ne soit pas seulement celle du mathématicien $»$ (id.).

Les redéfinitions du rôle de l'École, du rôle de l'éducateur et de l'articulation pensée/action sont mises en avant : l'école comme société parfaite pour devenir "autres que nous sommes », précisera Bachelard ; l'éducateur et le pédagogue définis comme des consciences de transformation; et la place des connaissances associant la pensée et l'action. Ainsi, l'action de l'homme pourra s'avérer décisive face à la révolution de la civilisation et permettre alors à l'Histoire d'être porteuse d'espoirs et d'espérances :

«Telle est l'importance individuelle et sociale du problème, celle qui nous faisait dire qu'il est au fond un problème de civilisation, ou plutôt d'adaptation de notre système éducatif, de sa structure, de son esprit et de ses méthodes, aux conditions nouvelles de la civilisation. C'est à la façon plus ou moins humaine dont on le résoudra que nous jugerons de l'espérance que porte l'histoire [...]»(Gal, 1950a, p. 49).

\section{Pédagogie et science(s)}

L'approche de la pédagogie dans ce contexte se trouve particulièrement bousculée par l'évolution 
des sciences, et en premier lieu, de la psychologie. Selon Roger Gal (1906-1966), conseiller technique de l'enseignement du second degré, ce « retentissement profond » est lié aux divers champs abordés dorénavant par la psychologie : la psychologie de l'enfant, l'étude de son développement, de la formation de ses fonctions motrices et mentales, de la naissance jusqu'à l'état adulte (Gal, 1950b, p. 160). La psychologie promet d'appréhender à nouveaux frais la réalité du psychisme de l'enfant en permettant de rendre compte de la diversité des âges et des différentes réalités auxquelles cela peut renvoyer. Elle met en avant qu'il existe des enfants, et non un enfant, et que l'enfance est un «perpétuel devenir ${ }^{26}$. En cela, la pédagogie, et en l'occurrence l'approche des situations d'apprentissage et d'enseignement, ne peuvent que s'en trouver questionnées.

Au sujet des tests de psychologie par exemple, Gal reconnaît leur utilité pour « situer l'avance ou le retard de l'individu »; par le même temps, ce dernier estime que ces tests ne constituent pas « le seul moyen, ni même le plus intéressant, pour mieux connaître l'enfant ». Il semble très indécis par la suite: "Sans doute il faudrait se garder d'employer mécaniquement n'importe quelle méthode [...]. Les tests ont leurs limites comme tout procédé [...] [mais] si l'éducation, nous dit Gal, suppose deux termes, l'adulte et l'enfant, elle ne saurait être éclairée sans une connaissance précise de l'enfant " (ibid., p. 163). Néanmoins, Gal est attentif aux risques en jeu par rapport aux sciences : "Il est permis de redouter la puissance d'action que ces sciences donnent sur l'homme, leur mauvaise utilisation au profit de toutes les tyrannies ou simplement d'un dogmatisme scientifique » (ibid., p. 160). La science, donc, permettrait d'« aller de l'empirisme hasardeux à une action toujours plus consciente de ce [que l'humanité] veut », cependant, conformément au style de pensée que j'ai commencé à définir, elle ne doit pas rendre impossible l'intention humaine : « n'acceptons pas que l'on asservisse les fins humaines à ce qui ne constitue jamais que des instruments de l'action, mais n'ignorons pas ces apports » (id.). La conception de Gal des relations entre pédagogie et sciences est très significative des Cahiers, en ce qu'elle semble reposer sur une conception non-négociable de ce qu'il appelle la possibilité de l'action dans le sens de l'humain. Ce raisonnement est sous-tendu par une conception nouvelle de la posture de l'enseignant. Sa fonction est en effet singulière d'un point de vue épistémologique. En gardant à l'esprit l'influence nouvelle de la psychologie, il faut considérer que le professeur n'est plus seulement un enseignant, mais un éducateur (Debesse, 1950, p. 153-158) : « l'attitude de l'éducateur, s'il utilise la psychologie, reste différente de celle du psychologue » (Gal, 1950b, p. 163). L'éducateur ne se substitue pas au scientifique, mais l'éducateur ne s'abandonne pas non plus à ce dernier. Gal juge que la mission de l'éducateur consiste à « faire mentir les tests ou plutôt de remettre incessamment en question leurs résultats et à chercher sans cesse un procédé pour éveiller, développer et former en l'enfant ce qui ne l'avait pas été jusque-là » (id.). La coexistence de ces deux idées, « la primauté de la pédagogie » et un rapport particulier de l'éducateur à la science, est particulièrement significative du style de pensée des Cahiers concernant par exemple la formation des enseignants et suggère, si l'on en croit Roger Gal, une alternative aux idées énoncées ci-dessous :

- l'éducation est conçue comme un art, ce qui confère à toute connaissance psychologique ou technique, le statut d'inutilité pour former un éducateur ;

- pour enseigner, la condition sine qua non est « la connaissance parfaite de ce qu'on doit enseigner $»(\mathrm{Gal}, 1950 \mathrm{~b}, \mathrm{p} .160)^{27}$.

Pour les Cahiers, la formation ${ }^{28}$ paraît indissociable de l'idée que l'éducateur doive expérimenter, explorer, participer à une dynamique collective autour de l'éducation et de la société. Les sillons d'un «plan d'ensemble » sont dessinés, comme le sousentendait Rossello en conclusion de sa contribution : «Libre à chacun de saisir la conjoncture favorable, de la dédaigner, ou même, si le cœur lui en dit, de ramer contre le courant "(Rossello, 1950, p. 9). Étant donné que les notions d'espace, de temps et de milieu ont été bouleversées par le progrès technique (Gal, 1950b, p. 160), le style de pensée à l'œuvre ouvre la possibilité d'un espace d'expérimentations et d'exploration depuis ces trois dimensions : l'espace; le temps; le milieu.

Dewey, une ouvre incontournable pour penser le pédagogue et la problématisation?

Nous pouvons nous demander si Dewey (18591952) n'est pas un penseur incontournable pour saisir la spécificité du style de pensée étudié. Un 
certain nombre d'indices incitent à concevoir l'œuvre de Dewey comme particulièrement éclairante pour saisir la spécificité du « collectif de pensée » étudié. À ce titre, signalons que Marie-Anne Carroi (18981976), traductrice de Dewey, contribue régulièrement à la revue et que les références à Dewey sont repérables chez des auteurs comme Weiler (1950) et Goblot (1950). Jean Bourjade (1883-1947), une des figures majeures ayant rendu possible cette histoire des Cahiers, voit en Dewey un «partisan décidé de l'éducation indirecte et des méthodes actives », mais également comme quelqu'un «qui [a] condamné avec une sévérité particulière les méthodes autoritaires et didactiques comme facteurs de désagrégation et de désintégration psychiques » (Bourjade, 1942, p. 25).

Pour reprendre l'objet de notre étude et cette question du positionnement de l'éducateur par rapport à la science, l'éducateur apparaît ici comme pris tout entier dans une " philosophie de l'action", formulation qu'utilise Weiler dans Les Cahiers au sujet de Dewey (1950b, p. 165), plutôt que déterminé par un cadre théorique issu par exemple de la psychologie.

On peut considérer que la problématisation novatrice des Cahiers pourrait se montrer en réalité très proche du «naturalisme empirique » de Dewey qui tient compte de "l'aspect social [et] physique de l'univers et se fonde sur « la valeur de vie comme expérience progressive et continue » (id.). En effet, le principe d'interaction chez Dewey, en tant que nous sommes toujours stimulés par un environnement, incite à se demander si le besoin d'exploration et d'investigation de l'homme n'est pas, à la fois, ce qui caractérise une "attitude scientifique de l'éducateur ${ }^{29}$ chez le militant des Cahiers (à partir de rapports complexes à la science) et l'enjeu d'une autre conception du cadre éducatif (conception pragmatique articulant principes philosophiques et expérimentations didactiques). En effet, lorsque Weiler résume cette idée de cette manière, " tout doit favoriser l'expérience individuelle et collective : le climat qui règne dans le milieu scolaire, le plan d'études, d'équipement » (id.), on voit bien que cette perception du climat induit le positionnement et la réflexion de l'éducateur :

«L'éducation apparaît de ce fait comme une suite de problèmes posés aux maîtres : quelle organisation et quelles activités paraissent adaptées le mieux possible aux divers degrés de développement des élèves; quelle part doit-on laisse à l'autorité et à l'expérience des adultes, aux enseignements du passé, aux livres et spécialement aux manuels; quels problèmes retenir pour exercer l'esprit d'observation, le jugement, l'expérience déjà acquise ; quels critères adopter pour retenir les expériences utiles à chacun ?» (id.)

Cette manière de poser les problèmes et cette vision commune des questions éducatives, propres à l'évolution du style de pensée dans la revue, se marginalisent pourtant peu à peu pendant la décennie des années 1960. Paradoxalement, alors que 1967 consacre l'institutionnalisation des sciences de l'éducation et que 1968 témoigne de la « rémanence » de quelques idées des classes nouvelles, le collectif de pensée est définitivement fragilisé par les départs en retraite et les disparitions de nombreux militants ayant animé les réseaux en question. D’autres réseaux se développent, d'autres logiques rentrent en ligne de compte, d'autres personnes prolongent alors l'aventure de la revue et du mouvement pédagogique.

\section{DISCUSSION}

Nous avons souhaité aborder la question des rapports entre science et pédagogie dans les Cahiers. Avant d'envisager de valider, ou non, l'hypothèse durkheimienne, il convient de bien mettre en évidence le souci de mettre en valeur certaines formulations au sein des échanges du collectif de pensée.

La particularité d'un collectif de pensée est de participer à un domaine de connaissance. Nul doute qu'ici que la publication de la revue ait contribué de manière complexe au champ de la pédagogie et indirectement à l'émergence des sciences de l'éducation ${ }^{30}$. La particularité d'un collectif de pensée est également de relier des individus ayant leur propre rattachement à des causes ou institutions : on observe ainsi les enjeux en marge de la revue (la réforme de l'institution scolaire, les questions d'ordre politique, l'émergence d'une discipline scientifique, l'organisation de la recherche pédagogique ${ }^{31}$ ) dans les échanges entre contributeurs (Roger Gal, Maurice Debesse, sont des exemples intéressants). Pour autant, un style de pensée caractérise ces échanges et il renvoie à la figure de l'enseignant des classes nouvelles.

En préface de l'ouvrage de Lefèvre (1949), Gustave Monod fournit les indications pour bien 
saisir une des figures possibles de l'enseignant des classes nouvelles en évoquant « des hommes jeunes d'esprit, souvent des maîtres déjà qualifiés par une longue pratique de leur métier, des hommes d'expérience et de foi » (Lefèvre, 1950, p. V). Dans cette volonté d'observation psychologique et cette ambition de partir de la connaissance de l'enfant, il explique qu'il faut surtout saisir l'importance de l'idée de «mise à l'épreuve d'instruments »:

« [...] dans les Classes Nouvelles, il s'agit moins d'une rénovation totale que d'une tentative pour conférer leur pleine efficacité à des méthodes déjà anciennes pour la plupart. Dans cet effort, il se peut qu'on rencontre chemin faisant des difficultés, des obstacles. Quand l'expérience les a révélés, $M$. Lefèvre ne les dissimule pas. Il sait que l'application des méthodes actives n'est pas celle d'une doctrine ou d'un système imposé par la voie administrative, mais bien plutôt la mise à l'épreuve d'un instrument qu'on peut modifier et assouplir à l'occasion, l'intérêt des enfants restant la seule règle absolue à observer. Cela exige assurément beaucoup de patience, de conscience, de loyauté, d'intelligence, d'esprit critique, de docilité à recueillir et à utiliser la leçon des faits. Ce sont les qualités qu'on trouvera dans ce livre » (ibid., p. VI).

Lorsque François Goblot signe en novembre 1949 la recension de cet ouvrage, il insiste sur la nature du livre, dont l'intérêt est de présenter « le bilan des efforts » produits par une équipe :

«Ce n'est ni un manuel de psychologie, ni un traité des méthodes actives, c'est le bilan des efforts qu'a fait un chef d'équipe de $6^{\mathrm{e}}$ nouvelle en $1945-46$ pour mettre la psychologie au service de l'éducation et de l'enseignement » (1949, p. 83).

Il apparaît que la pertinence de la démarche de l'équipe de Lefèvre repose sur l'élaboration d'une expérience et des leçons à tirer à partir des faits observés :

« [...] Il s'agit plus modestement de voir comment un maître a été amené de plus en plus à s'orienter vers des préoccupations psychologiques dans une classe nouvelle, comment il a adapté son enseignement à ces préoccupations et quelles suggestions lui a inspirées son expérience » (Lefèvre, 1949, p. 9).

\section{Les partages conceptuels durkheimiens}

La délimitation effectuée par Durkheim pour distinguer la réflexion pédagogique de l'éducation comme chose sociale et de la science de l'éducation est apparue particulièrement éclairante. En effet, ces enseignants militants énoncent indéniablement des réflexions pédagogiques, au sens où ils mobilisent des connaissances diverses (histoire, sociologie, philosophie) au sujet de l'éducation pour prendre de la distance sur les faits observés concernant leur quotidien. Le mouvement émergeant autour de la revue représente même, d'une certaine manière, une ébauche originale de la science de l'éducation, telle que l'envisageait Durkheim lui-même. Le format de la revue a joué ici un rôle très important. Historiquement, la revue constitue un espace entre science de l'éducation et expérimentations locales. Les colonnes de la revue servent à rendre compte des expérimentations des classes nouvelles, mais très vite, la revue publie les prises de notes des stages de Sèvres, puis des articles contenant des connaissances très spécifiques.

Cependant, les partages conceptuels de Durkheim ont leur limite en tant que grille de lecture du chantier historique mobilisé. Si Durkheim nous permet de comprendre ce que peut être une réflexion pédagogique, il apparaît difficile d'en déduire une posture liée à la pratique. La question devient la suivante : quelle posture d'ordre pratique peut être reconstruite dans le sillage du jugement émis sur la routine? En étant quelque peu caricatural, nous estimons que Durkheim envisage, d'une certaine manière, une pédagogie sans pédagogues. Il définit la pédagogie avec sérieux et noblesse. Pour autant, Durkheim n'est pas tendre avec les pédagogues qu'il mentionne: Rousseau, Pestalozzi notamment. Durkheim disparaissant en 1917, sa méfiance pour les pédagogues n'est-elle pas structurelle, mais conjoncturelle?

\section{Limites des partages conceptuels durkheimien comme grille de lecture}

Les propos de Durkheim dans Éducation et sociologie présentent la particularité d'être publiés initialement en 1922. Cependant, les quatre études composant cet ouvrage proviennent des cours promulgués par Durkheim ; ces textes ont été réunis grâce à un de ses élèves, Paul Fauconnet, mais il faut préciser 
que la publication est postérieure à la disparition de Durkheim en 1917. Maurice Debesse, auteur de la préface de cet ouvrage, insiste sur l'idée selon laquelle, l'utilité des propos de Durkheim dépend fortement de la prise en compte de son contexte historique (Durkheim, 2006, p. 6). En effet, les propos fortement critiques de Durkheim envers la forme spéculative de la science de l'éducation à la fin du XIX ${ }^{e}$ siècle, la philosophie de l'éducation artificialiste et les pédagogues historiques comme Rousseau ou Pestalozzi n'est intelligible que si l'historicité de ces positions est prise en compte. À l'encontre de ces trois réalités institutionnelles ou intellectuelles, Durkheim adresse un reproche commun : ne pas prendre en compte l'« existant », ignorer systématiquement les conditions d'existence de l'éducation.

« La science de l'éducation» est officiellement une chaire d'enseignement qui apparaît en 1883 (Gautherin, 2002). Ce sont principalement des philosophes qui ont alors la charge de ces cours. Pour Durkheim, cette science de l'éducation a l'inconvénient d'être très spéculative et les disciplines scientifiques qui pourraient l'empêcher de sombrer dans cette direction ne sont pas encore assez développées. Au sujet de la philosophie de l'éducation, Durkheim reproche à certains auteurs de se méprendre sur ce qu'est l'éducation concrètement. Ainsi, l'observation socio-historique prouve selon lui que le rôle de l'éducation à travers les âges est la socialisation méthodique des jeunes générations. Enfin, si Durkheim s'irrite des propos de Rousseau, c'est parce que le pédagogue ne s'appuie pas sur les faits, ne prend pas appui sur les réalités et préfère inventer à nouveaux frais un système aventureux et irréalisable.

Néanmoins, Fauconnet souligne combien la pensée de Durkheim conçoit d'une manière parfaitement claire l'idée d'une science de l'éducation, ainsi qu'une conception idéaliste de la pédagogique, tant qu'elle ne bascule pas dans l'utopie. Dès lors, le célèbre concept durkheimien de "théorie-pratique " devient symétriquement essentiel pour saisir la position de Durkheim sur la distinction entre « science de l'éducation » et «pédagogie » et dans le même temps, proposer une lecture du quotidien des premiers contributeurs des Cahiers. S'ils permettent de bien saisir ce qui permet aux pédagogues d'émettre un jugement au sujet de pratiques routinières et sclérosées et de mettre en jeu la science par rapport à l'évolution du monde, les partages conceptuels durkheimiens ont leur limite pour décrire le militantisme pédagogique : ils ne peuvent décrire plus précisément la démarche du pédagogue par rapport à son action pragmatique ${ }^{32}$ autour de la question de l'étude du milieu au nom d'une exigence d'humanité dans la société ${ }^{33}$.

\section{L'ouvre de Rousseau comme point de rupture}

Pour François Goblot (1950), Rousseau n'est pas un dangereux utopiste, il est avant tout un des penseurs de l'Éducation nouvelle, dont le contexte éducatif est l'éducation privée, le préceptorat. C'est le cas de Rabelais, Montaigne. Pour Goblot, une seconde étape dans l'histoire de l'Éducation nouvelle se produit au XIX ${ }^{e}$ siècle avec la création d'écoles privées; Pestalozzi en est le plus illustre représentant. Mais Goblot explique qu'une troisième période s'est ouverte autour de l'idée d'une éducation nationale en lien avec l'éducation nouvelle. Or, Goblot fait commencer cette période au XX $\mathrm{X}^{\mathrm{e}}$ siècle, en mobilisant des références et des contextes que Durkheim n'a pu connaître.

Rousseau apparaît comme une figure importante, principalement depuis l'idée de dissociation des volontés à l'œuvre dans la célèbre « leçon de choses ». Cette posture tient pour radicale la distinction du savoir du maître et de l'apprentissage de l'enfant, en vue de le préserver de la "société corrompue ". C'est le point nodal de la pensée des Cahiers. Mais, l'obstacle qui se présente pour les Cahiers est que la proposition rousseauiste est intenable dans le contexte institutionnel de la revue (à quoi ressemblerait un système éducatif articulé autour de l'idée que les savoirs livresques n'apparaîtraient que lorsque les élèves fêteraient leur douzième ou treizième anniversaire?). Les Cahiers se préoccupent du second degré et le problème n'est pas simplement la confrontation aux choses, mais devient la confrontation aux signes : l'écriture, les œuvres, la culture, les machines. La question des militants devient : quelle posture et quels postulats permettraient d'articuler le principe de la leçon de choses et la culture?

Les apports de la filiation lyonnaise (de la critique de l'institution scolaire au champ de l'expérience) pour penser le pédagogue militant

Entre Durkheim et les débuts des Cahiers se situent deux œuvres fondamentales pour saisir la 
spécificité de la figure du pédagogue par rapport à la science et la question de la militance pédagogique: les œuvres d'Edmond Goblot et de Jean Bourjade.

\section{La critique de l'institution scolaire}

Rappelons-nous la filiation lyonnaise du collectif de pensée des Cahiers. Le professeur Edmond Goblot (1858-1935), philosophe lyonnais et père de François Goblot, démontre en 1925 que le diplôme du baccalauréat est à la fois, "barrière et niveau » (Goblot, E., 2010, p. 72) et que son unique fonction est de maintenir et de légitimer une distinction entre classes sociales. À cette occasion, il dénonce le fonctionnement de l'enseignement secondaire, qu'il s'agisse des contenus et des méthodes pédagogiques, avec une centration stratégique sur la question du latin, pour maintenir la domination d'une classe sociale. Alors que la sociologie durkheimienne permettait de décrire l'éducation comme étant « la socialisation méthodique des jeunes générations » et de justifier le rôle et la responsabilité de l'État dans le développement d'une institution spécifique consacrée à cette mission de socialisation (l'École), l'essai sociologique de Goblot critique une logique focalisée sur la distinction des classes, anticipant en cela sur la sociologie britannique du curriculum et les critiques sociologiques françaises de la reproduction et de l'école capitaliste. Dès lors, puisque les processus de distinction sont des constructions, aboutissant à des hiérarchies illégitimes, la figure de l'enseignant se retrouve confrontée à sa propre image dans un jeu de miroir faisant apparaître son influence au sein de la classe et, à une autre échelle, sa participation au fonctionnement de l'institution scolaire. Les jugements de valeur à l'œuvre, les méthodes pédagogiques et les curriculae scolaires sont donc pré-déterminés par un type de société et d'institutions.

\section{Science de l'éducation lyonnaise et alternative au didactisme}

À cette critique se greffe l'émergence d'une science de l'éducation lyonnaise, dont Bourjade constitue une figure importante. C'est l'émergence de la notion d'expérience, dans l'alternative qu'elle propose à ce que l'on entend par didactisme à cette époque, qui va nous intéresser ici. Bourjade entend par didactisme
« la seule forme convenable de la transmission des connaissances dans les sociétés autoritaires et traditionnalistes » (1942, p. 4). Le didactisme a pour nature « d'isoler l'œuvre de l'ouvrier, le résultat de son élaboration, de figer l'opération active en structure statique, autrement dit de ne s'intéresser qu'au savoir comme tel, pris absolument, au détriment de celui qui apprend » (ibid., p. 6). Si Bourjade explique que le développement de l'intérêt pour l'enfance coïncide avec « le discrédit de la conception objectiviste et réaliste de la connaissance ", « le triomphe de l'esprit d'investigation sur l'esprit de codification » et à « la pénétration de l'esprit expérimental dans le domaine pédagogique » (id.). Néanmoins, reconnaît-il, on ne peut éliminer définitivement le didactisme, car l'accélération du progrès induit une telle croissance des connaissances et une telle complexification des aptitudes pour saisir au mieux l'évolution de la civilisation qu'il restera des personnes favorables au didactisme. Bourjade estime que Rousseau, en tant que "véritable créateur de la science de l'enfant », « [...] qui le premier en a isolé l'objet et fixé les perspectives » se situe à l'opposé du didactisme. Avec Rousseau, se dessine l'émergence d'une théorie de l'éducation négative couplée avec une éducation indirecte, « la première se donnant pour tâche de préserver des interventions inopportunes le dynamisme interne de la croissance, la seconde de faire du milieu extérieur le champ d'expérience le plus instructif » (ibid., p. 9).

Lorsque Bourjade souligne que l'activité psychique se manifeste sous un triple aspect : « la volonté qui en est la propulsion, le sentiment qui en est le retentissement intime et en marque la valeur pratique et l'intérêt, la connaissance qui en est l'instrument $»$ (ibid., p. 24) ; c'est l'œuvre de Dewey qui apparaît finalement :

« Dewey professe que l'esprit est inséparable de son environnement et que, par conséquent, ses fonctions d'observation, d'intégration mnémonique, d'imagination, de coordination conceptuelle et de liaison logique ne se constituent à travers l'enfance et n'accomplissent leur œuvre propre que sous la pression des problèmes posés par la vie à l'être tout entier dans le milieu physique et le milieu social » (id.).

Bourjade en tire donc la conclusion suivante :

« Un rapport de stricte réciprocité unit donc, dans une telle doctrine, la théorie de l'être psychique comme 
organisme indivis à la théorie de l'expérience comme organisation constructive. Dès lors l'éducation perd son caractère institutionnel arbitraire : elle est la croissance de l'expérience et la réalisation du moi chez l'individu au sein de l'univers et de la société grâce à quoi se maintient et se tourne en progrès la continuité de la civilisation» (ibid., p. 24-25).

\section{CONCLUSION}

L'enseignant militant des Cahiers présente un profil caractéristique : souvent agrégé, il formule bien des réflexions pédagogiques, au sens de Durkheim, mais ces discours s'articulent autour d'expériences, au sens de Dewey. Le cadre étudié autour de cette figure d'enseignant-militant délimite une science de l'éducation particulière (et officieuse) où le scientifique coopère avec le praticien, dans un contexte d'adaptation à des réalités économiques et industrielles, tout en maintenant un impératif humaniste et démocratique. La figure épistémologique du pédagogue n'est dès lors plus condamnée à la spéculation sans lendemains.

Si ce contexte est loin derrière nous ${ }^{34}$, nul doute que la stratégie de formation et de vulgarisation (publications) pourrait être utile aux actions menées actuellement, notamment dans le cadre de l'Institut Français d'Éducation. Alors que ces expérimentations reposent sur un principe d' « ingénieries didactiques coopératives »(Sensevy, 2011, p. 739), au sein desquelles professeurs, formateurs et chercheurs prennent place, sans hiérarchie a priori, l'expérience de ces classes nouvelles pourrait alors redevenir utile. 


\section{NOTES}

1. Dans une conférence de presse en décembre 1948, Monod décline les principes directeurs des Classes Nouvelles : « adapter étroitement l'enseignement au degré de développement mental de l'enfant » et « partir du monde où il vit, retrouver les représentations qu'il s'en fait et ne pas substituer notre vision d'adulte, nos conceptions abstraites et constantes de l'univers où ne pénètrent guère que sensations et images » (cité par Chapoulie, 2010, p. 366). C'est en vertu de ces principes que diverses innovations ont été pensées, envisagées depuis le cadre expérimental des classes nouvelles : étude du milieu ; travail en équipe des élèves; recours à des enquêtes et à des recherches individuelles.

2. L'usage de la notion de "problématisation » renvoie aux travaux de Dubreucq au sujet de l'éducation républicaine, et en particulier à son angle de recherche portant sur l'idée de «sphère de pensée ». L'appartenance à un même régime de discours, une même "sphère de pensée », n'empêche pas les divergences et l'émergence de « système de tensions $»$ (Dubreucq, 2004, p. 7-8).

3. André Robert pose alors plusieurs questions à ces deux intervenants avec, comme arrière-plan, la question de la pensée pédagogique.

4. Pour Alain Marchive, « ce n'est sans doute pas un hasard si Brousseau a pensé donner le nom d'épistémologie expérimentale à la recherche en didactique des mathématiques ». Marchive poursuit : « [...] si la préoccupation praxéologique et la volonté d'amélioration des pratiques d'enseignement n'est pas absente (dans le développement les ingénieries didactiques en particulier), son projet est d'abord scientifique et vise à proposer un cadre explicatif (la théorie des situations didactiques) qui permette de comprendre le fonctionnement des systèmes didactiques » (2008, p. 67).

5. La question de la spécificité d'un « fait social » est abordée dans Les règles de la méthode sociologique (Durkheim, 2010).

6. Il est intéressant de signaler la réticence marquée de Durkheim envers Rousseau. À plusieurs reprises dans Education et sociologie, Durkheim mentionne Rousseau dans des propos très critiques. Au sujet des questions éducatives, le sociologue n'hésite pas à douter de l'application des idées rousseauistes : "Nous plaindrions des enfants auxquels on appliquerait rigoureusement la méthode de Rousseau ou celle de Pestalozzi » (Durkheim, 2006, p. 88). L'applicabilité des théories rousseauistes depuis le politique ne convainc pas davantage Durkheim. Ainsi, ce dernier déclare-t-il : « Rousseau eût été vraisemblablement aussi mauvais ministre que mauvais éduca- teur» (Durkheim, 2006, p. 78). La méfiance de Durkheim s'exerce envers les pédagogues comme Rousseau ou Pestalozzi, non envers la pédagogie en général.

7. Nous retrouvons en cela la division et la complémentarité imaginée par Kant entre les écoles « normales » et les écoles « expérimentales».

8. Sur cette notion de «monde de pensée », voir aussi : Go, H. L. (2012). Retour sur un paradoxe de la normativité éducative.

9. Pour Klein, « la correspondance de Binet met à jour son projet comme l'objet d'aucune des sciences particulières de l'époque » (Klein, 2008, p. 26). L'étude de cette correspondance renvoie à de la non-science, de l'idéologie, à des pratiques politiques et/ou sociales.

10. Le travail de Klein s'inscrit dans le prolongement des recherches engagées par Serge Nicolas autour « [des] documents d'archives permettant de retracer avec précision les parcours et les destins d'acteurs célèbres ou oubliés de la psychologie française, mais également des revues, institutionnalisations et conceptualisations qui participèrent à l'histoire de la discipline à l'échelle internationale » (Klein, 2011, p. 13-14).

11. « Auprès de M. Monod, directeur de l'Enseignement du Second degré, les problèmes des classes nouvelles étaient surtout étudiés par le Conseil technique pédagogique : Alfred Weiler, Roger Gal, Claire Roby, Georges Petit. Il siégeait rue de Grenelle, à l'étage de la Direction du Second degré, dans un petit bureau que l'on trouvait, à condition de connaître, au fond d'un couloir obscur. Là, tous les problèmes personnels et généraux étaient discutés ; là, on reconstruisait chaque jour le monde et, par la même occasion, l'Université » (Goblot, 1968, p. 29).

12. Centre Universitaire d'Information Pédagogique.

13. Institut Pédagogique National.

14. Par exemple, les différentes contributions de Suzanne Brunet semblent être des exemples pertinents.

15. Il faut noter que ces préoccupations s'inscrivent dans un contexte où les pensées éducatives de Dewey, Decroly et Freinet ont une forte notoriété.

16. Tourisme et enseignement. In : Les Cahiers pédagogiques pour l'enseignement du Second degré, 7, 12-18.

17. Il faut souligner le rôle important qu'a joué Pol-Simon, à la fois, dans le mouvement des classes nouvelles er en particulier, dans la création du Lycée Marseilleveyre.

18. Sont alors concernés quatre adultes et vingt enfants (18 filles et 6 garçons), qui sont élèves de Troisième, Seconde et Première, avec une grande proportion d'élèves hellénistes. Le voyage a duré une quinzaine de jours dont 3 jours à l'aller et 3 jours au retour, pour faire le voyage en bateau. 
19. Le voyage scolaire permet à chacun de se découvrir. «[... Nous avons eu avec nos élèves des contacts que nous n'aurions pas eus sans cela $[\ldots] »$ (ibid., p. 14). « [...] Nous avons découvert nos élèves et nos élèves nous ont découverts $[\ldots] »(i d$.$) .$

20. La Déclaration Universelle des Droits de l'Homme, la Seconde Guerre Mondiale, le problème noir, la faim dans le monde, l'enfance dans le monde, l'effort artistique français sont des thématiques évoquées dans ce cours.

21. Ce plan fut pensé par la commission Langevin-Wallon, dont les origines professionnelles variées étaient une des caractéristiques (Riondet, 2010).

22. Bureau International d'Education.

23. Plusieurs auteurs comme Jean Fourastié et Alfred Sauvy, que Jean-Michel Chapoulie définirait comme « les nouveaux experts des sciences sociales » (Chapoulie, 2006), interviennent dans la revue dans les années 50 et 60.

24. Il s'agit de la formulation de Bachelard que l'on peut lire dans la revue.

25. Alors que l'habitude est finalement de l'ordre d'une "rémanence ", au sens du « maintien d'attitudes dont la finalité première est oubliée » (Bugnard, 2006, p. 312).

26. « [...] la psychologie dit exactement que l'être de l'enfant et de l'adolescent est en un perpétuel devenir, où le passé, le présent et l'avenir sont en fait toujours enveloppés, où l'aspiration à être mieux, à grandir, est le ferment constant du progrès et donc de l'éducation » (Gal, 1950b, p. 161).

27. Pour Gal, un enseignant doit pouvoir s'appuyer sur un bagage de connaissances (psychologiques notamment) qui ne se restreint pas à son champ d'expertise dans un domaine d'enseignement. Ce qui place la pédagogie en dehors de l'alternative entre art et savoirs liés à une discipline. Pour Durkheim, la pédagogie correspond à une attitude mentale intermédiaire qui n'est ni de l'art, ni de la science (Durkheim, 2006).

28. Pour Maurice Debesse (professeur de psychologie et de pédagogie à la faculté de lettres de Strasbourg), trois éléments doivent composer la formation professionnelle des enseignants du Second degré : les connaissances spéciales propres à la discipline qu'il doit enseigner, les ferments d'une culture l'aidant à comprendre son temps, une initiation psychologique et pédagogique.

29. Nous reprenons une formulation de Weiler (1950b).

30. Les sciences de l'éducation sont institutionnalisées en 1967, notamment à Bordeaux (Jean Château), à la Sorbonne (Maurice Debesse) et à Caen (Gaston Mialaret). Ce trio provient « d'une psychologie qui se veut expérimentale, tournée vers la pédagogie, proche de l'éducation nouvelle (Houssaye, 2009, p. 172). Il est intéressant de souligner que si Les Cahiers ont participé aux échanges liées aux questions de réforme et d'épistémologie qui ont sous-tendu la création de la discipline, ils n'ont pas véritablement commenté en direct cet événement de 1967 dans leurs colonnes.

31. L'histoire des Cahiers fluctue également au rythme complexe de l'histoire de la recherche pédagogique, et en particulier l'émergence de l'IP. (puis INRDP et enfin INRP) et du CRD., dont le fondateur est un certain Louis Cros.

32. François Goblot, créateur de la revue en 1945, énonce les contours de ses réflexions pédagogiques et la démarche spécifique de l'éducateur : coordonner les enseignements et se coordonner ; avoir, en tant qu'enseignant, « le souci de manifester [aux yeux des élèves] l'unité de la science et de la vie » (Goblot, 1946, p. 4) ; faire converger les enseignements sur un même objet ; mobiliser certains principes de l'Education nouvelle (réalisme, activité, intérêt, individualisation du travail) en vertu des objectifs envisagés ; et " toujours faire preuve d'imagination » (Goblot, 1945b, p. 7).

33. L'ambition est d'élever l'humanité, écrit Monod dans une circulaire du 20 août 1945, « [...] c'est-à-dire de développer en chacun tout ce qui peut faire de lui un homme éclairé capable de se dégager de sa profession, de son parti ou de sa secte, pour juger librement son temps et le juger à la lumière des leçons du passé, aussi bien que des exigences de l'avenir » (cité par Goblot, 1946, p. 1).

34. Notamment le contexte de l'expérimentation des classes nouvelles (1945-1952). 


\section{RÉFÉRENCES}

Astolfi, J.-P. et Houssaye, J. (1996). Didactique et pédagogie sont dans un bateau... Éducations, 18-21.

Bachelard, G. (1950). Philosophie et pédagogie au $\mathrm{XX}^{\mathrm{e}}$ siècle. Les Cahiers pédagogiques pour l'enseignement du Second degré, 3-4, 28-31.

Ballon J., Delannoy J. et Labernede M. (1959). Pour ou contre l'actualité. Les Cahiers pédagogiques pour l'enseignement du Second degré, 12, 31-32.

Blais, M.-C., Gauchet, M. et Ottavi, D. (2002). Pour une philosophie politique de l'éducation. Paris : Éditions Bayard.

Bloch, M.-A. (1953). Pédagogie des classes nouvelles. Paris : Presses Universitaires de France.

Bois L., Chavanne P. et Merignon H. (1958). Tourisme et enseignement. Les Cahiers pédagogiques pour l'enseignement du Second degré, 7, 12-18.

Bouisseren, Y (1959). L'éducation de la liberté. Les Cahiers pédagogiques pour l'enseignement du Second degré, 12, 61-62.

Bourjade, J. (1942). Lintelligence et la pensée de l'enfant. Paris : Presses Universitaires de France.

Bugnard, P.-P. (2006). Le temps des espaces pédagogiques. De la cathédrale orientée à la capitale occidentée. Nancy : Presses Universitaires de Nancy.

Carroi, M.-A. (1950). Dewey. Les Cahiers pédagogiques pour l'enseignement du Second degré, 3, 177-180.

Chambon, J., Delchet, R. et Lefèvre, L. (1974). Anthologie des pédagogues français contemporains. Paris : Presses Universitaires de France.

Chapoulie, J.-M. (2006). Les nouveaux spécialistes des sciences sociales comme « experts » de la politique scolaire en France 1945-1962. Genèses, 64, 124-145.

Chapoulie, J.-M. (2010). L'École d'État conquiert la France. Rennes: Presses Universitaires de Rennes.

Chavanne, P. (1959). Dans le cadre de la classe. Les Cahiers pédagogiques pour l'enseignement du Second degré, 12, 40-41.

Condette, J.-F. et Savoye, A. (2011). Le congrès du Havre (31 mai - 4 juin 1936) : Albert Châtelet et la réforme de l'enseignement du Second degré. Carrefours de l'éducation, 31, 61-88.

Congrès de Marseille (1959). Le comportement de l'élève à l'école. Les Cahiers pédagogiques pour l'enseignement du Second degré, 12, 38.

Debesse, M. (1950). Psychologie et réforme de l'éducation. Les Cahiers pédagogiques pour l'enseignement du Second degré, 3, 153-158.

Delannoy, J. (1959). Léducation pratique ne suffit pas. Les Cahiers pédagogiques pour l'enseignement du Second degré, $12,45$.

Dewey, J. (1947/1938). Expérience et éducation. (Trad. M.-A. Carroi). Paris : Bourrelier / Colin.

Douglas, M. (1999/1986). Comment pensent les institutions. (Trad. A. Abeillé). Paris : La Découverte.

Dubreucq, E. (2004). Une éducation républicaine. Paris : Vrin.
Durkheim, E. (2006/1922). Éducation et sociologie. Paris : Presses Universitaires de France.

Durkheim, E. (2010/1975). Les règles de la méthode sociologique. Paris : Éditions Flammarion.

Éducation et prospective. Animateurs et chercheurs en éducation. Saint-Yorre. : Éditions Éducation et prospective.

Fleck, J. (2005/1935). Genèse et développement d’un fait scientifique. (Trad. J. Gibelin). Paris : Les Belles Lettres.

Fabre, M. (2011). Éduquer pour un monde problématique. Paris : Presses Universitaires de France.

Foucault, M. (1971). Lordre du discours. Paris : Éditions Gallimard.

Fourastié, J. (1950). L'évolution économique contemporaine et la formation des jeunes générations. Les Cahiers pédagogiques pour l'enseignement du Second degré, 3/4, 10-18.

Gal, R. (1950a). Orientation et sélection. Les Cahiers pédagogiques pour l'enseignement du Second degré, 3/4, 4349.

Gal, R. (1950b). La psychologie au service de l'éducateur. Les Cahiers pédagogiques pour l'enseignement du Second degré, 3, 159-163.

Gautherin, J. (2002). Une discipline pour la République. La Science de l'éducation en France (1882-1914). Bern : Éditions Peter Lang.

Go, H. L. (2012). Retour sur un paradoxe de la normativité éducative. Nantes : Recherches en éducation, 14.

Goblot, E. (2010/1925). La barrière et le niveau. Paris : Presses Universitaires de France.

Goblot, F. (1945a). Université et mouvements de jeunesse. Esprit, 11, 587-594.

Goblot, F. (1945b). Méthodes nouvelles dans l'enseignement du Second degré. Les Dossiers pédagogiques pour l'enseignement du Second degré, 1, 1-7.

Goblot, F. (1946). Les sixièmes nouvelles. Les Dossiers pédagogiques pour l'enseignement du Second degré, 5, 1-8.

Goblot, F. (1950). Quelques réflexions. Les Cahiers pédagogiques pour l'enseignement du Second degré, 3, 185-190.

Goblot, F. (1968). Le mouvement pédagogique des classes nouvelles. Les Cahiers pédagogiques, 78, 29-31.

Houssaye, J. (2009). De la naissance des philosophes de l'éducation en France. In : Vergnoux, A. (Éd.), 40 ans des sciences de l'éducation. L'âge de la maturité ? Questions vives. Actes de colloque de Caen (20-22 février 2007) (165-178). Caen : Presses Universitaires de Caen.

Hubert, R. (1950). Éducation et tradition. Les Cahiers pédagogiques pour l'enseignement du Second degré, 3/4, 26-28.

Klein, A. (2008). Correspondance d'Alfred Binet. Jean Larguier des Bancels. Nancy: Presses Universitaires de Nancy.

Klein, A. (2011). Correspondance d'Alfred Binet. Lémergence de la psychologie scientifique (1884-1911). Nancy : Presses Universitaires de Nancy.

Lefèvre, L. (1949). Le professeur psychologue. Paris : Presses Universitaires de France.

Lefèvre, L. (1958). Une aide précieuse pour la géographie. 
Les Cahiers pédagogiques pour l'enseignement du Second degré, 4, 68-69.

Legrand, G. (1959). Écouter battre le cour du monde. Les Cahiers pédagogiques pour l'enseignement du Second degré, 12, 88-95.

Les Cahiers pédagogiques (1972). Quelle est l'orientation des Cahiers? Les Cahiers pédagogiques, hors-série, 2-3.

Marchive, A. (2008). La pédagogie à l'épreuve de la didactique. Rennes: Presses Universitaires de Rennes.

Massat, A. (1958). La méthode active et concrète. Les Cahiers pédagogiques pour l'enseignement du Second degré, 4, 59-62.

Masset, C. (1958). Érudition et culture. Les Cahiers pédagogiques pour l'enseignement du Second degré, 4, 36-39.

Monod, G. (1950). Libres opinions. Les Cahiers pédagogiques pour l'enseignement du Second degré, 3/4, 3-5.

Moraze, C. (1950). Humanisme nouveau, éducation nouvelle. Les Cahiers pédagogiques pour l'enseignement du Second degré, 3/4, 19-22.

Pol-Simon, R. et Goblot, F. (1947). Les classes nouvelles. Bulletin de l'ANECNES, 8, 13-16.

Riondet, X. (2010). L'Éducation nouvelle et la question de l'autorité. In E. Prairat (dir.), L'autorité éducative : déclin, érosion ou métamorphose? (87-106). Nancy : Presses Universitaires de Nancy.
Robert, A. (2001). Débat : Denis Kambouchner/Philippe Meirieu, Revue Française de Pédagogie, 137, 5-16.

Rossello, P. (1950). La crise de croissance de l'enseignement du second degré. Les Cahiers pédagogiques pour l'enseignement du Second degré, 3/4, 7-9.

Savoye, A. (2010). Réforme pédagogique, réforme disciplinaire : l'expérience des Classes nouvelles dans l'enseignement du second degré (1945-1952). In R. d'Enfert et P. Kahn (dir.), En attendant la réforme (51-64). Grenoble: Presses Universitaires de Grenoble.

Sensevy, G. (2011). Le sens du savoir. Bruxelles : De Boeck. Weiler, A. (1949). Introduction à l'étude du milieu. Les Cahiers pédagogiques pour l'enseignement du Second degré, 1, 8-10.

Weiler, A. (1950a). L'humaniste du temps présent. Les Cahiers pédagogiques pour l'enseignement du Second degré, 3/4, 23-24.

Weiler, A. (1950b). Les variations et l'avenir de l'éducation nouvelle. Les Cahiers pédagogiques pour l'enseignement du Second degré, 3, 164-168.

Westphal, G. (1964). Un travail d'archives. Les Cahiers pédagogiques pour l'enseignement du Second degré, 3, 174-178. 